\title{
ESTRATÉGIAS ADOTADAS NO ENFRENTAMENTO DA COVID-19: RELATO DE EXPERIÊNCIA PROFISSIONAL
}

\author{
Paulo Henrique da Cruz Ferreira ${ }^{1}$ \\ Liliane da Consolação Campos Ribeiro² \\ Gessiane de Fátima Gomes ${ }^{1}$ \\ Renata Patricia Fonseca Gonçalves ${ }^{2}$
}

\begin{abstract}
https://orcid.org/0000-0003-1828-8914 https://orcid.org/0000-0003-1828-8914 https://orcid.org/0000-0002-2872-1155 http://orcid.org/0000-0002-5292-2053
\end{abstract}

Objetivo: relatar a experiência acerca da estruturação das ações adotadas no enfrentamento a pandemia da Covid-19. Método: estudo descritivo, do tipo relato de experiência, tendo como referência o Método do Arco de Charlez Maguerez. A experiência foi vivenciada por líderes que compõem a equipe de enfrentamento à Covid-19, durante os meses de fevereiro a abril de 2020, em um hospital de médio porte, referência macrorregional de média e alta complexidade do Vale do Jequitinhonha em Minas Gerais. Resultados: descreveu-se a implantação das práticas recomendadas ao combate à disseminação do novo Coronavírus baseadas em cinco eixos a saber: revisão dos fluxos operacionais de serviço, educação na saúde como estratégia de enfrentamento à pandemia, ampliação dos leitos e das equipes para atendimento da Covid-19 e saúde mental dos profissionais durante a pandemia. Considerações Finais: as estratégias adotadas para o enfrentamento da Covid-19 na instituição permitiram que as orientações definidas pela Organização Mundial de Saúde e Ministério da Saúde fossem implementadas de acordo com a realidade local. Enfatiza-se diante da pandemia a relevância da manutenção de contínua atualização técnico científica.

Descritores: Infecções por Coronavírus; Pandemias; Planejamento Operacional; Prevenção e Controle; Riscos Ocupacionais.

\section{STRATEGIES ADOPTED IN COPING WITH THE COVID-19: REPORT OF PROFESSIONAL EXPERIENCE}

Objective: to report the experience about the structuring of actions adopted to cope with the Covid-19 pandemic. Method: descriptive study, of experience-report type, taking as a reference the Arch Method, of Charlez Maguerez. The experience was lived by leaders who make up the team to cope with the Covid-19, during the months of February to April 2020, in a medium-sized hospital, macro-regional reference of medium and high complexity in Vale do Jequitinhonha in Minas Gerais. Results: the implementation of best practices to combat the spread of the new Coronavirus was described based on five axes, namely: review of operational flows of service, health education as a strategy to cope with the pandemic, expansion of beds and the team to meet the Covid-19 and mental health professionals during the pandemic. Final Consideratioins: strategies for coping with the Covid-19 in the institution allowed implementing the guidelines set by the World Health Organization and the Ministry of Health in accordance with the local reality. Before the pandemic, the ongoing maintenance of scientific-technical update is extremely relevant.

Descriptors: Infections caused by Coronaviruses; Pandemics; Operational Planning; Prevention and Control; Occupational Risks.

\section{LAS ESTRATEGIAS ADOPTADAS PARA HACER FRENTE A LA COVID-19: INFORME DE EXPERIENCIA PROFESIONAL}

Objetivo: reportar la experiencia acerca de la estructuración de las medidas adoptadas para el enfrentamiento de la pandemia de Covid-19. Metodo: estudio descriptivo, del tipo informe de experiencia, tomando como referencia el Método del Arco, de Charlez Maguerez. La experiencia fue vivenciada por los líderes que conforman el equipo para enfrentar el Covid-19, durante los meses de febrero a abril de 2020, en un hospital de tamaño medio, referencia macro-regional de mediana y alta complejidad del Vale do Jequitinhonha en Minas Gerais. Resultados: se describió la aplicación de las mejores prácticas para combatir la propagación del nuevo Coronavirus basada en cinco ejes, a saber: revisión de flujos operacionales de servicio, educación en salud como estrategia para enfrentar la pandemia, la expansión de las camas y el equipo para el atendimiento de la Covid-19 y los profesionales de la salud mental durante la pandemia. Consideraciones Finales: las estrategias para enfrentar la Covid-19 en la institución permitió la implantación de los lineamientos establecidos por la Organización Mundial de la Salud y el Ministerio de Salud en conformidad con la realidad local. Se destaca en el rostro de la pandemia, la pertinencia del mantenimiento permanente de actualización técnica y científica.

Descriptores: Infecciones Causadas por Coronavirus; Pandemias; Planificación Operacional; Prevención y control; Riesgos Ocupacionales.

'Santa Casa de Caridade de Diamantina, MG

${ }^{2}$ Departamento de Enfermagem da Universidade Federal dos Vales do Jequitinhonha e Mucuri, MG. Autor Correspondente Renata Patricia Fonseca Gonçalves E-mail: renata.fonseca@ufvjm.edu.br Recebido: 10/5/2020 Aceito: 09/6/2020 


\section{INTRODUÇÃO}

A Covid-19 é uma doença infecciosa causada pelo vírus Severe Acute Respiratory Syndrome Coronavirus 2 (SARS-CoV-2), popularmente chamado de novo Coronavírus, que causa manifestações clínicas leves como a de um resfriado ou, em casos mais graves, evoluem para síndrome de desconforto respiratório e possue necessidade de cuidados em unidades de terapia intensiva ${ }^{1-2}$.

Embora estudos iniciais realizados na China relatem uma ligação entre o mercado de animais selvagens e a maioria dos casos de infecção, indicando uma possivel transmissão de animal para humano, dados epidemiológicos atuais têm demonstrado que há uma rápida transmissão de SARS-CoV-2 de humano para humano, por meio de gotículas ou contato, o que propiciou efetivamente a sua disseminação mundial ${ }^{1,3}$.

Em 11 de março de 2020, a Organização Mundial de Saúde (OMS) caracterizou como estado de pandemia a Covid-19, o que representa um dos maiores desafios sanitários em escala mundial desse século ${ }^{4}$. O novo Coronavírus levou a uma mudança abrupta nas rotinas dos serviços de saúde, observando-se um cenário de intensificação de internações hospitalares, superlotação de unidades, falta de leitos e de equipamentos, impactando, também, a saúde das equipes de saúde assistenciais ${ }^{5-6}$.

Esse novo contexto impôs aos gestores o estabelecimento de estratégias de reestruturação do espaço físico, fluxos de atendimento, garantia de suprimentos em meio a uma terrivel pandemia, dentre outras inúmeras demandas. Concomitantemente, o dimensionamento adequado da equipe frente a inúmeros afastamentos trazem à tona um grande desafio para as instituições de saúde em preservar e zelar pelo exercício profissional, de forma segura e conforme as recomendações sanitárias e garantias legais?

Nessa perspectiva, para que a manutenção do tratamento de pacientes infectados não se torne um risco para a saúde ocupacional, é imperativo chamar atenção para falhas na biossegurança dos trabalhadores, que tem sido a realidade observada em diversos países ${ }^{8}$.

A contaminação e o adoecimento dos profissionais envolvidos no atendimento aos pacientes é uma realidade. Como observado na China, em que foram registrados 3.387 casos de trabalhadores da saúde acometidos pela Covid-19 e levaram a 22 mortes 9 . No Brasil, segundo o relatório do Comitê Gestor de Crise Covid-19 do Conselho Federal de Enfermagem (Cofen), há mais de 5.000 casos de trabalhadores dessa categoria com diagnóstico confirmado da doença, 145 óbitos e mais de 10 mil casos em investigação ${ }^{10}$.

Em meio a tantos desafios impostos pela Covid-19, a educação na saúde também se faz necessária como estratégia de enfrentamento à pandemia, pois o novo cotidiano requer que os profissionais também revisem suas competências, habilidades e atitudes e adequem-nas ao momento atual para o desenvolvimento do cuidado, compreendendo que esse se desenvolve em niveis de complexidade distintos, porém indissociáveis, que transitam desde uma simples ação educativa sobre a biossegurança, até as práticas de cuidado na alta complexidade 6

Considerando que a pandemia associada ao SARS-CoV-2 expôs os serviços de saúde a um novo cenário epidemiológico e à necessidade de formulação de planos para respostas rápidas à disseminação da doença, neste trabalho, se propôs relatar a experiência acerca da estruturação das ações adotadas no enfrentamento da pandemia da Covid-19.

\section{MÉTODO}

\section{Tipo de Estudo}

Trata-se de um estudo descritivo do tipo relato de experiência. A base metodológica utilizada foi a problematizadora, tendo como referência o Método do Arco de Charlez Maguerez ${ }^{11}$, por possibilitar a participação ativa dos profissionais, colocando-os não como meros receptores, mas como fonte de conhecimento e experiências, envolvendo-os na discussão e engajando-os na identificação e solução de problemas dos seus cotidianos.

\section{Cenário do Estudo}

O local do estudo foi uma instituição hospitalar que conta com um total 125 leitos, aproximadamente 400 profissionais e oferece atendimento a pacientes particulares, de convênios médicos e do Sistema Único de Saúde (SUS). É referência macrorregional de média e alta complexidade, atendendo a sede e mais de 30 municípios do Vale do Jequitinhonha, em Minas Gerais. No momento atual, este serviço de saúde é referência para tratamento da Covid-19.

\section{Período de realização da experiência}

O presente estudo delimita-se na experiência profissional vivenciada nos meses de fevereiro a abril do ano de 2020.

\section{Sujeitos envolvidos na experiência}

O relato foi baseado na experiência de líderes dos setores estratégicos da instituição como Núcleo de Segurança do Paciente, Serviço de Controle de Infecção Hospitalar, Segurança e Medicina do trabalho e Coordenação de Enfermagem, que compõem a equipe de enfrentamento à Covid-19.

\section{Aspectos Éticos}

$O$ estudo cumpriu com todas as exigências que regem as pesquisas com seres humanos. A respeito disso, este projeto foi submetido e aprovado pelo Comitê de Ética em Pesquisa institucionalizado, recebendo parecer sob o no 4.023.342/2020. 


\section{OBJETIVOS DA EXPERIÊNCIA}

Estruturar as ações para o enfrentamento da pandemia da Covid-19 em um hospital de referência no Vale do Jequitinhonha Minas Gerais.

\section{DESCRIÇÃO DA EXPERIÊNCIA}

O enfrentamento à pandemia da Covid-19 exige esforços de diferentes setores, atores e instituições da sociedade, reunidos em um amplo escopo de ações articuladas, especialmente de âmbito econômico, social e sanitário.

No campo da saúde, faz-se necessário ações em dois âmbitos: (1) macropolítico, principalmente na garantia de leitos hospitalares, respiradores e medidas farmacológicas; e (2) práticas educativas e ações de cuidado pautadas em tecnologias leves, caracterizadas pelas normas, protocolos e fluxos de atendimento ${ }^{12}$

Baseado nas ações articuladas à saúde, a alta gestão do referido hospital definiu por meio de portaria interna uma equipe composta por líderes dos setores estratégicos e instituiu a equipe de enfrentamento à Covid-19.

O objetivo dessa equipe foi a implantação das melhores práticas recomendadas ao combate à disseminação da infecção, considerando a realidade local e as diretrizes publicadas pela OMS e Ministério da Saúde (MS).

Assim, a equipe de enfrentamento à Covid-19 definiu como eixos norteadores institucionais as ações relatadas a seguir.

\section{Revisão dos fluxos operacionais de serviço}

Com o início da pandemia, as principais preocupações apresentadas pelos profissionais foram relacionadas aos trabalhadores do grupo de risco e ao fluxo de atendimento ao paciente que apresenta suspeita ou confirmação da doença. Este primeiro contato com o paciente ainda gera dúvidas e apreensões, principalmente devido ao elevado número de informações, mudanças constantes nas recomendações e fake news disponibilizado pelas mídias sociais.

O MS recomenda o fluxo rápido, priorizando o atendimento no momento da chegada e estabelecendo um nível de prioridade, tendo a disposição um médico, um enfermeiro e um técnico de enfermagem para a assistência, assim como a área exclusiva também é tida como uma recomendação absoluta e indispensável ${ }^{12}$.

Seguindo as recomendações do MS, os setores da instituição foram redefinidos para as internações dos pacientes suspeitos ou confirmados de acordo com o avanço da pandemia nesta região de saúde. Os fluxos institucionais ao atendimento para casos suspeitos ou confirmados da Covid-19 foram amplamente discutidos, adaptados para a realidade e, inicialmente, dá-se por meio da classificação de risco e seque até o desfecho final de alta ou óbito, a depender da gravidade dos sintomas apresentados pelo paciente.

Como uma das ações implementadas, trabalham na linha de frente de atendimento à Covid-19 apenas os profissionais que não se enquadram como grupo de risco. Os colaboradores foram orientados a apresentarem atestado médico relatando a situação de saúde que o caracterize como grupo de risco e, gradativamente, foram realocados em outros setores da instituição ou liberados para gozo de férias.

Como medida de vigilância, diariamente é realizada uma ligação telefônica para todos os profissionais que tenham contato com caso suspeito ou confirmado da Covid-19, buscando sinais e sintomas precoces de síndrome respiratória e, caso o colaborador apresente alguma queixa, é orientado a procurar o pronto atendimento da instituição para realização de teste rápido e notificação.

Todas as ações criadas especificadamente para este enfrentamento deram origem a um manual interno denominado "Manual de enfrentamento da Covid-19" que busca facilitar a tomada de decisão pelos colaboradores. Por ser um hospital referência à outras instituições hospitalares menores, o manual foi disponibilizado no formato online para toda macrorregião, com o objetivo de servir como uma linha guia no desenvolvimento das ações contra o Coronavírus.

\section{A educação na saúde como estratégia de enfrentamento à pandemia}

O acelerado processo de atualização do conhecimento acerca da Covid-19 exige que os profissionais que estão na linha de frente de atendimento, estejam atualizados de forma permanente e aptos a desenvolverem uma prática de cuidados que seja segura para o paciente, para si próprio, para os demais membros da equipe que atuam no cenário e para a comunidade por onde esse profissional irá circular após o término de seu turno de trabalho ${ }^{5}$.

Nesse contexto, a educação na saúde revela-se como uma importante estratégia de enfrentamento à pandemia, pois consiste na produção de conhecimentos relativos à formação e ao desenvolvimento do indivíduo para a atuação profissional nos serviços de saúde, a fim de torná-los aptos a atuarem de maneira a garantir a integralidade do cuidado, a segurança deles próprios como trabalhadores e dos usuários e a resolubilidade do sistema ${ }^{11}$.

Partindo do pressuposto que o serviço de saúde deve capacitar todos os seus profissionais e acreditando que, mesmo durante a pandemia, a educação na saúde é uma ferramenta imprescindivel, adotou-se, na instituição, estratégia de capacitação individual e em pequenos grupos.

Em um primeiro momento, foram abordadas com a equipe questões referentes à pandemia com dados epidemiológicos, inferências sobre as possibilidades do surgimento desta nova 
patologia na região, caracterização clínica com os principais sinais e sintomas, modos de transmissão, transmissibilidade comunitária, prevenção da Covid-19 intra-hospitalar e aspectos relevantes ao isolamento social.

Consideramos que houve uma satisfatória integração entre o conhecimento e as experiências dos colaboradores envolvidos, proporcionando um momento de transformação e ressignificação profissional, visto que, além da aprendizagem, houve ganhos imensuráveis quanto à cooperação, à empatia e ao comprometimento da equipe.

Como segunda etapa da educação na saúde, o Procedimento Operacional Padrão (POP) sobre o uso de Equipamento de Proteção Individual (EPI) foi revisado em reunião ordinária da equipe de enfrentamento da Covid-19, oportunizando um momento de alinhamento das diversas informações inerentes a temática proposta e adaptando-se à realidade da instituição. Foi criado um folder de fácil visualização sobre o passo a passo de paramentação e amplamente divulgado nas mídias eletrônicas internas.

Uma das medidas propostas pelo grupo foi a criação de multiplicadores para treinamento individual do profissional. Desta forma, enfermeiros, técnicos de enfermagem e de segurança do trabalho, com o apoio docente do curso de graduação em enfermagem da Universidade Federal dos Vales do Jequitinhonha e Mucuri realizaram inúmeras simulações realísticas abordando a paramentação e retirada correta dos EPI na assistência ao paciente com suspeita ou diagnóstico da Covid-19. Todos os profissionais da equipe multiprofissional tiveram a oportunidade de praticar sob a orientação e o olhar atento dos multiplicadores responsáveis por aquele turno ou setor. Destaca-se que durante a simulação medidas de prevenção foram observadas como o distanciamento e uso de máscaras.

Ao término das capacitações práticas setoriais foi criada nova estratégia com o intuito de aperfeiçoar o exercício de paramentação, além de acolher individualmente os profissionais que retornavam do período de férias ou afastamentos. Foi implementada, assim, uma estação de treinamento diário em local pré-estabelecido e com horário fixo, estando sempre disponivel um multiplicador dotado de um kit de EPI.

Além do objetivo inicial, essa capacitação prática permeou outros desígnios conforme a demanda e especificidades dos setores, como adaptação de rotinas na central de material e esterilização e auditoria de prontuário, adequação na segregação dos resíduos dos serviços de saúde, revisão dos POPs para o transporte interno do paciente suspeito ou confirmado da Covid-19, coleta de exames bioquímicos e diagnósticos, bem como a higienização concorrente e terminal do ambiente e dos artigos hospitalares.
Destaca-se que, para a implementação da capacitação teórico-prático, foram adotadas metodologias ativas, prevendo processos de apropriação e produção do conhecimento ${ }^{13}$. O processo de aprendizagem utilizou-se de instrumentos pedagógicos promotores de habilidades-competências e simulações no contexto da própria prática profissional, a qual desafiou os sujeitos envolvidos a um processo dialético de apropriação e transformação da realidade.

Por fim, cabe ressaltar que as mídias sociais foram utilizadas como meio para a difusão e atualização do conhecimento. Conforme o mundo se reinventava cientificamente e as condutas frente à Covid-19 eram revisadas, os grupos profissionais de WhatsApp foram altamente utilizados para postagem de notas e protocolos internos ou também para oferta de cursos online gratuitos em plataformas digitais.

Ampliação dos leitos e equipe para atendimento da Covid-19

Apesar da recessão econômica que o país vivencia neste momento, o enfrentamento da pandemia na instituição tem sido realizado com governabilidade e economicidade, garantindo a segurança dos profissionais e pacientes.

Uma ala de enfermaria com 12 leitos foi preparada e equipada para a internação especifica de pacientes com suspeita ou confirmação do novo Coronavírus, denominada "Clínica de Internação Covid-19", além da realocação de ambientes do pronto atendimento e Unidade de Terapia Intensiva, atualmente com 20 leitos adultos. Uma equipe multiprofissional foi contratada em caráter temporário para que essa unidade e outros profissionais foram selecionados em processo seletivo e estão em stand-by para contratação a medida que for necessário.

\section{Saúde mental dos profissionais durante a pandemia}

Outro aspecto que merece atenção é o elemento emocional das equipes de saúde. Frente à pandemia, os profissionais têm demonstrando ansiedade devido à possibilidade iminente de exposição ao vírus e ao adoecimento, que pode ter um curso bastante desfavorável e, ainda, possivel transmissão aos familiares.

Nesse sentido, sabendo que o autocontrole no trabalho pode evidenciar menores niveis de estresse ocupacional ${ }^{14}$ gerou uma demanda por acompanhamento psicológico aos colaboradores que demonstravam abalos emocionais. Solicitamos auxílio aos psicólogos da cidade para que, voluntariamente, pudessem realizar este acolhimento e atendimento aos profissionais, a fim de oferecer recursos para a compreensão do momento e auxiliar nas estratégias de enfrentamento.

A psicóloga da instituição se responsabilizou por receber as demandas individuais e direcionou-as ao psicólogo volun- 
tário, que realizou o atendimento e acompanhamento online.

Esse apoio ofertado pela instituição está de acordo com a OMS que, recentemente, publicou um guia para orientar cuidados a saúde mental de diversos grupos, incluindo profissionais de saúde. Para os trabalhadores da saúde, o estresse e a pressão de lidar com o seu trabalho, acrescido do risco de adoecer, provocam severos problemas de saúde mental, aumentando o turnouver e a síndrome de Burnout ${ }^{14-15}$.

\section{PRINCIPAIS RESULTADOS ALCANÇADOS}

A criação de uma frente de enfrentamento à Covid-19 e a implementação de eixos que nortearam as ações na instituição permitiu atender às recomendações de combate ao novo Coronavírus emanadas pela OMS e pelo MS. No entanto, mais do que o alcance das recomendações dos órgãos legislativos, essa experiência propiciou aos profissionais de saúde discussões necessárias a respeito de medidas que melhorem as condições de trabalho durante a pandemia, assim como promoveu capacitações para o reconhecimento dos riscos e aquisição de comportamento seguro.

As atividades implementadas permitiram, também, a condução dos sujeitos ao alcance de novas estruturas em seu desenvolvimento e, ainda, à mobilização do que aprenderam na realização de novas tarefas, tendo condições de contextualizarem com seus conhecimentos prévios.

\section{LIMITAÇÕES PARA A EXPERIÊNCIA}

Nesse cenário de pandemia, as estratégias educativas têm sido utilizadas para levar orientações a um maior número de individuos, incluindo os profissionais que estão na linha de frente dos serviços de saúde. No entanto, a constante mudança no referencial teórico relacionado ao tema e, consequentemente, nas recomendações de combate à pandemia foi o principal fator limitador. Somado a isso, o acelerado processo de disseminação de notícias falsas, fake news, gera confusão entre os profissionais e um descrédito em relação às orientações dos órgãos de saúde e institucional.

\section{Contribuições para A Prática}

A experiência relatada possibilitou dialogar e problematizar as questões do enfrentamento da pandemia, organizando em eixos norteadores as condutas dentro de uma instituição hospitalar referência para o atendimento ao paciente com Covid-19.

Foi possivel sistematizar o cuidado e, ao mesmo tempo, instigar o pensamento crítico reflexivo em relação à necessidade da proteção individual e coletiva, focado na assistência ao paciente que se encontrava em precaução por contato e respiratória, em um cenário que o colaborador está temeroso pela nova situação.

Contribuiu também para despertar nos colaboradores a importância de se utilizar a precaução padrão na assistência diária, prática negligenciada na rotina assistencial e que contribui para a ocorrência de infecções cruzadas entre os pacientes e contaminação da própria equipe assistencial.

Este foi um momento de grande construção, uma vez que muitos colaboradores realizaram valorosas contribuições, permitindo que as ações fossem aprimoradas e facilitadas para todos. O compartilhamento de saberes proporcionou a edificação de uma rotina que permitiu a ressignificação na elaboração do enfrentamento da pandemia, em que todos os envolvidos no processo tiveram oportunidade de ser atuantes nessa estruturação, compreendendo que a autonomia, o diálogo e a participação ativa e conjunta são essenciais para a construção coletiva do conhecimento e transformação efetiva da realidade.

Apesar da multiplicidade de sentimentos, medo, ansiedade e insegurança pelo novo cenário que enfrentamos, observamos grande envolvimento dos profissionais e recebemos um feedback positivo de todas as equipes em relação às capacitações, tanto teórica quanto prática. Percebemos com clareza que, a estruturação de ações para o enfrentamento à Covid-19 e sua ampla divulgação, trouxe aos colaboradores segurança para o desempenho de suas atividades assistenciais.

\section{CONSIDERAÇÕES FINAIS}

As estratégias adotadas para o enfrentamento da Covid-19 na instituição permitiram que as orientações definidas pelo MS e OMS fossem implementadas de acordo com a realidade local.

Tendo em vista que o novo Coronavírus foi recentemente descoberto e que devido a isso há uma constante mudança no referencial teórico relacionado ao tema, enfatiza-se a relevância da manutenção de contínua atualização técnico científica.

Almejamos que diante de tantas mudanças, os profissionais de saúde e, em especial os da enfermagem, permaneçam como protagonistas de seu cotidiano, buscando aperfeiçoamento de suas ações, ansiando sempre por uma assistência segura para si e para seus pacientes.

Destaca-se que o compartilhamento de experiências nos campos de saúde representa importante estratégia para agregar conhecimento e ampliar o olhar para as possibilidades de combate à epidemia no contexto tão novo para todos.

Contribuição dos Autores: PHCF: contribuiu com a concepção e desenho do estudo, análise e interpretação dos dados, redação do artigo, revisão crítica e revisão final; LCCR: contribuiu com a concepção e desenho do estudo, análise e 
interpretação dos dados, redação do artigo, revisão crítica e revisão final; GFG: contribuiu com a concepção e desenho do estudo, análise e interpretação dos dados, redação do artigo, revisão crítica e revisão final; RPFG: contribuiu com a concepção e desenho do estudo, análise e interpretação dos dados, redação do artigo, revisão crítica e revisão final.

\section{REFERÊNCIAS}

1. Huang C, Wang Y, Li X, Ren L, Zhao J, Hu Y et al. Clinical features of patients infected with 2019 novel coronavirus in Wuhan, China. Lancet [Internet]. 2020 [cited 2020 apr 29]; 395 (10223). Available from: https://www.thelancet.com/ journals/lancet/article/PIISO140-6736(20)30183-5/fulltext

2. World Health Organization (WHO). Naming the coronavirus disease (COVID-19) and the virus that causes it. 2020 [cited 2020 apr 29]. Available from: http://who.int/emergencies/diseases/novel-coronavirus-2019/technicalguidance/naming-the-coronavirusdisease-(COVID-2019)-and-the-virus-that-causes-it

3. L Wang, Y Wang, D Ye, Q Liu. Review of the 2019 novel coronavirus (SARS-CoV-2) based on crente evidence. International Journal of Antimicrobial Agents [Internet]. 2020 [cited 2020 apr 29]; 12 (43). Available from: https://doi. org/10.1016/j.ijantimicag.2020.105948

4. Barreto ML, Barros ALD, Carvalho MS, Codeço CT, Hallal PRC, Medronho RA, et al. O que é urgente e necessário para subsidiar as políticas de enfrentamento da pandemia de COVID-19 no Brasil? Revista Brasileira de Epidemiologia [Internet]. 2020 [cited 2020 jun 03]; 23: e200032. Disponivel em: https://doi.org/10.1590/1980-549720200032

5. Rodrigues NH, Silva LGA. Gestão da pandemia Coronavirus em um hospital: relato de experiência profissional. $\mathrm{J}$. nurs. Health [Internet]. 2020 [cited 2020 apr 29]; 10(n.esp.): e20104004. Available from: http://docs.bvsalud.org/ biblioref/2020/05/1095608/2-gestao-da-pandemia-coronavirus-em-um-hospital-relato-de-expe_r8ZHcz8.pdf

6. Ramos RS. A Enfermagem Oncológica no Enfrentamento da Pandemia de Covid-19: Reflexões e Recomendações para a Prática de Cuidado em Oncologia. Revista Brasileira de Cancerologia [Internet]. 2020 [cited 2020 may 08 ]; 66 (Tema Atual): e-1007. Available from: https://doi.org/10.32635/2176-9745.RBC.2020v66nTemaAtual.1007

7. World Health Organization. State of the world's nursing 2020: investing in education, jobs and leadership [Internet] Geneva: WHO; 2020 [acesso 2020 apr 23]. Available from: https://www.who.int/publications-detail/ nursingreport-2020

8. Fiho JMJ, Assunção AA, Algranti E, Garcia EG, Saito CA, Maeno M. A saúde do trabalhador e o enfrentamento da COVID-19. Rev. bras. saúde ocup. [Internet]. 2020 [cited 2020 may 10]; 45: el4. Available from: http://www.scielo.br/ scielo.php?script=sci_arttext\&pid=S0303-76572020000100100\&lng=en

9. Wang J, Zhou M, Liu F. Exploring the reasons for healthcare workers infected with novel coronavirus disease 2019 (COVID-19) in China. J Hosp Infect[Internet]. 2020 [cited 2020 apr 22]; 105:100-101. Available from: https://doi. org/10.1016/j.jhin.2020.03.002

10. Conselho Federal de Enfermagem [Internet]. Brasilia, DF: Cofen; 2020 [cited 2020 jun 04]. Available from: http:// observatoriodaenfermagem.cofen.gov.br

11. Falkenberg MB, Mendes TPL, Moraes EP, Souza EM. Educação em saúde e educação na saúde: conceitos e implicações para a saúde coletiva. Ciência \& Saúde Coletiva [Internet]. 2014 [cited 2020 apr 17]; 19(3): 847-52. Available from: https://doi.org/10.1590/1413-81232014193.01572013

12. Ministério da Saúde (BR). Secretaria de Vigilância em Saúde. Plano de Contingência Nacional para Infecção Humana pelo novo coronavírus COVID-19. [Internet] Brasília: Ministério da Saúde; 2020 [cited 2020 may 10]. Available from: https://portalarquivos2.saude.gov.br/images/pdf/2020/fevereiro/13/plano-contingencia-coronavirus-COVID19. pdf

13. Morais JT, Silva AE, Gontijo TL, Ribeiro RF, Faria RGS. Avaliação do impacto da capacitação no trabalho para o cuidado de pessoas com estomias. Enferm Foco [Internet]. 2019 [cited 2020 jun 02]; 10(3): 93-98. Available from: https://doi.org/10.21675/2357-707X.2019.v10.n3.1810

14. Rocha, RPS, Valim MD, Oliveira JLC, Ribeiro AC. Caracteristicas do trabalho e estresse ocupacional entre enfermeiros hospitalares. Enferm Foco [Internet]. 2020 [cited 2020 jun 02]; 10(5): 51-57. Available from: https://doi. org/10.21675/2357-707X.2019.v10.n5.2581

15. World Health Organization (WHO). Mental health and psychosocial considerations during the COVID-19 outbreak [Internet]. 2020 [cited 2020 apr 30]. Available from: https://www.who.int/docs/defaultsource/coronaviruse/mentalhealthconsiderations.pdf?sfvrsn=6d3578af_2 